\title{
Temporary extracorporeal left ventricular support with transapical ProtekDuo cannula
}

\author{
Matthew L. Goodwin, MD, ${ }^{a}$ Sophia Roberts, MD, ${ }^{\mathrm{a}, \mathrm{b}}$ Brent C. Lampert, DO, ${ }^{\mathrm{c}}$ and \\ Bryan A. Whitson, MD, PhD, ${ }^{\mathrm{a}}$ Columbus, Ohio
}

\footnotetext{
From the ${ }^{\mathrm{a}}$ Division of Cardiac Surgery, Department of Surgery and ${ }^{\mathrm{c}}$ Division of Cardiology, Department of Medicine, The Ohio State University; and ${ }^{\mathrm{b}}$ The Ohio State University College of Medicine, Columbus, Ohio. Abstract submitted for consideration to the AATS 2020 Resident Case Report Competition.

Disclosures: Dr Whitson is a consultant/speaker for Abbott Laboratories, is a proctor for Medtronic, and serves on the Clinical Events Committee of TransMedics Organ Care System. All other authors reported no conflicts of interest.

The Journal policy requires editors and reviewers to disclose conflicts of interest and to decline handling or reviewing manuscripts for which they may have a conflict of interest. The editors and reviewers of this article have no conflicts of interest.

Received for publication Nov 12, 2020; accepted for publication Nov 12, 2020; available ahead of print Nov 20, 2020.

Address for reprints: Bryan A. Whitson, MD, PhD, The Ohio State University Wexner Medical Center, Doan Hall N816, 410 W 10th Ave, Columbus, OH 43210 (E-mail: bryan.whitson@osumc.edu).

JTCVS Techniques 2021;5:76-9

2666-2507

Copyright (C) 2020 The Authors. Published by Elsevier Inc. on behalf of The American Association for Thoracic Surgery. This is an open access article under the CC BY-NC-ND license (http://creativecommons.org/licenses/bync-nd/4.0/).

https://doi.org/10.1016/j.xjtc.2020.11.004
}

Patients with advanced heart failure presenting with cardiogenic shock often require temporary mechanical circulatory support (tMCS) to preserve and recover end-organ function when medical therapy is inadequate. Extracorporeal membrane oxygenation (ECMO) is a modality of tMCS being used with increased frequency in these critically ill patients, specifically the Interagency Registry for Mechanically Assisted Circulatory Support (INTERMACS) profile 1 phenotype. ${ }^{1}$ Peripheral insertion of venoarterial ECMO is expeditious and can provide adequate hemodynamic support to allow for end-organ stabilization or recovery. Unfortunately, ECMO as tMCS in refractory cardiogenic shock is not without complications, including vascular access problems, bleeding, and worsening left ventricle (LV) distention and pulmonary edema. ${ }^{1}$ We present the following case of a patient with advanced heart failure with refractory cardiogenic shock in need of tMCS as a bridge to decision. A seldom-reported technique of temporary left-sided support using a dual-lumen, single-access site cannula was chosen in this patient with significant aortic valve pathology. This report has been deemed as exempt human research by The Ohio State University Office of Responsible Research Practices Institutional Review Board with waiver of need for consent.

\section{CASE REVIEW AND TECHNIQUE}

A 51-year-old male patient with a history of ischemic cardiomyopathy, heart failure with reduced ejection fraction (New York Heart Association IV, stage D), and coronary artery disease developed cardiogenic shock. He had undergone coronary artery bypass grafting 16 years ago

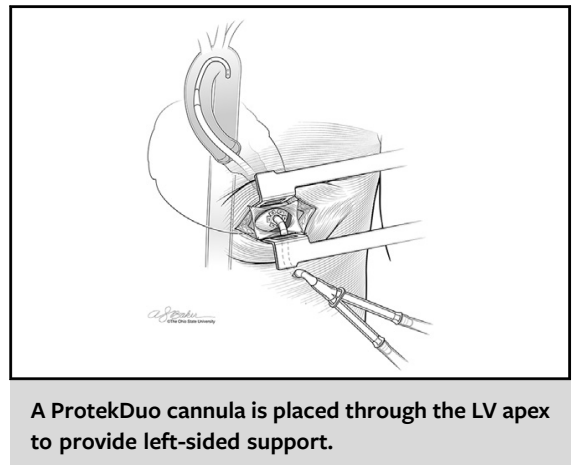

\section{CENTRAL MESSAGE \\ Temporary left-sided support using a dual-lumen, single-access site cannula provides excellent hemodynamics, end-organ re- covery, and mobility in patients with significant aortic valve pathology.}

See Commentary on page 80.

and repeat coronary artery bypass grafting 7 years thereafter. Echocardiography showed severely reduced left ventricular function with an ejection fraction of $10 \%$, moderate-to-severe aortic regurgitation (AR), and moderate aortic stenosis (Figure 1). He was initiated on dual-inotrope and diuretic therapies with initial improvement in endorgan function. On hospital day 3, he acutely decompensated with refractory hypotension associated with worsening renal function and rising lactate levels despite escalating medical management. We elected to insert a transapical ProtekDuo cannula (LivaNova PLC, London, United Kingdom) for temporary left-sided support due to his moderate-to-severe AR, as peripheral ECMO and other tMCS devices were contraindicated.

\section{Technique}

1. As our patient had acutely decompensated, was on multiple inotropes, in cardiogenic shock, INTERMACS level 1, and had previous cardiac surgery, we decided for acute temporary mechanical support to bridge to 

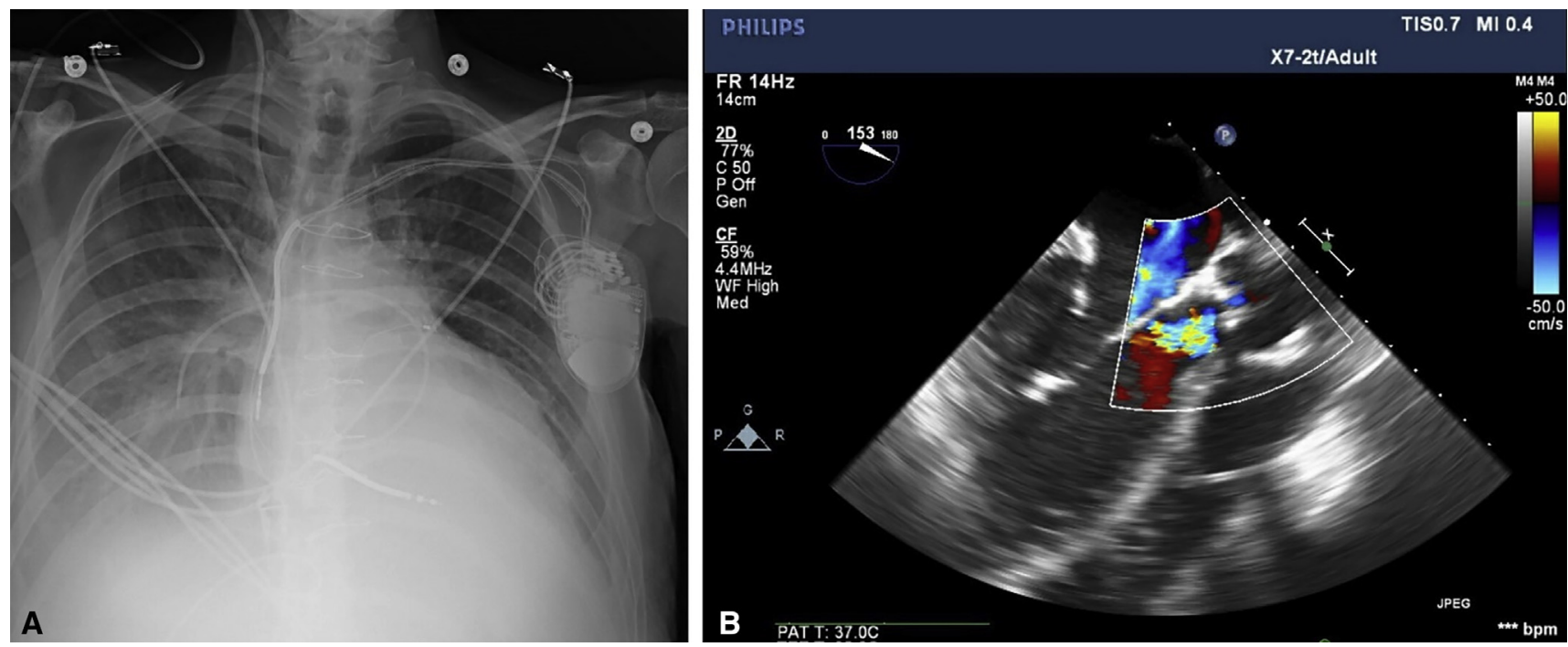

FIGURE 1. A, Preoperative chest radiograph showing cardiomegaly and increased pulmonary vascular markings. A pulmonary artery catheter is in place. Sternal wires are present from previous coronary revascularization. B, Preoperative transesophageal echocardiogram with color flow Doppler showing a tricuspid aortic valve with moderate-to-severe aortic insufficiency and moderate aortic stenosis.

decision of candidacy for durable left ventricular assist device. He was brought to the hybrid operating theater and a transesophageal echocardiography (TEE) probe was inserted.

2. We made a left anterolateral minithoracotomy in the fifth intercostal space. After the thoracotomy, we gained access to the LV by incising the pericardium overlying the LV apex. Three concentric \#2 pledgeted Ti-Cron sutures (Medtronic, Minneapolis, Minn) were placed around the dimple of the apex, avoiding the left anterior descending coronary artery, which was supplied by a patent vein graft on the preoperative angiogram. Intravenous systemic anticoagulation of unfractionated heparin was administered to ensure an activated clotting time in excess of 250 seconds.

3. Using TEE guidance, an 18-gauge introducer needle was placed into the left ventricular cavity through the pledgeted sutures in the apex. A 0.035 guidewire was advanced through the aortic valve and positioned in the descending thoracic aorta using fluoroscopy and TEE. The wire was inserted through the LV apex and across the valve into the descending thoracic aorta directly. There was no exchange catheter or additional wire used.

4. We employed a modified Seldinger technique to serially dilate the apex before advancing the 31-F ProtekDuo cannula through the intercostal space below the thoracotomy incision. The ProtekDuo was place in through the intercostal space below through a separate incision to facilitate hemostasis and secure closure of the thoracotomy and minimize any potential of cannula impingement. The cannula was positioned with the inflow in the LV and the outflow lumen in the descending thoracic aorta. The pledgeted Ti-Cron sutures were tightened and secured (Figure 2). Additional sutures and a Hollister horizontal drain attachment device (Hollister Inc, Libertyville, Ill) secured the cannula to the skin.

5. The cannula was deaired and connected to a CentriMag pump (Abbott, Abbott Park, Ill). The pump speed was increased under TEE guidance, and we evaluated the inflow port position in the LV cavity, LV decompression, and right ventricular function. The device had a flow of 4.5 liters per minute at $4100 \mathrm{rpm}$ with no $\mathrm{AV}$ opening and moderate residual AR. The residual AR did not contribute to any significant mixing, and the LV was well decompressed.

\section{DISCUSSION}

In this case, we describe the use of a single-site, duallumen ProtekDuo cannula inserted though the LV apex to provide temporary left-sided support in a patient designated as INTERMACS profile 1 . The cannula was placed through a left anterolateral minithoracotomy with the aid of TEE and fluoroscopy. It provided excellent hemodynamic support without hemolysis or insertion-site bleeding and allowed for end-organ recovery. The goal for this patient was a durable LVAD, as he had a recent history of smoking. We have found that the anticoagulation profile of CentriMag pumps and the Protek Duo (in right ventricular support applications) is very favorable. The activated partial thromboplastin time needed is low and adverse bleeding events and rates are very low. Hemolysis is negligible. The patient was progressing in the hospital, including ambulating and 

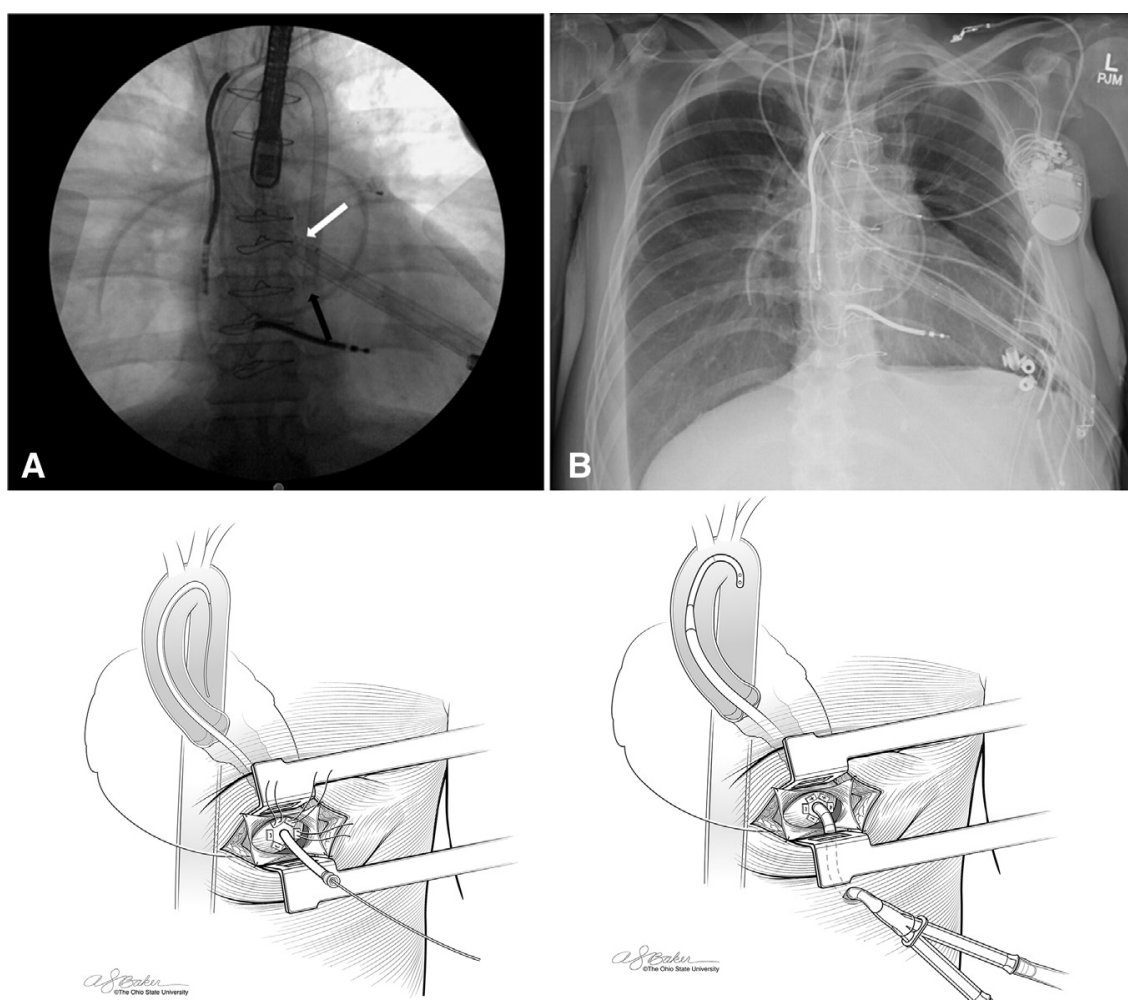

C

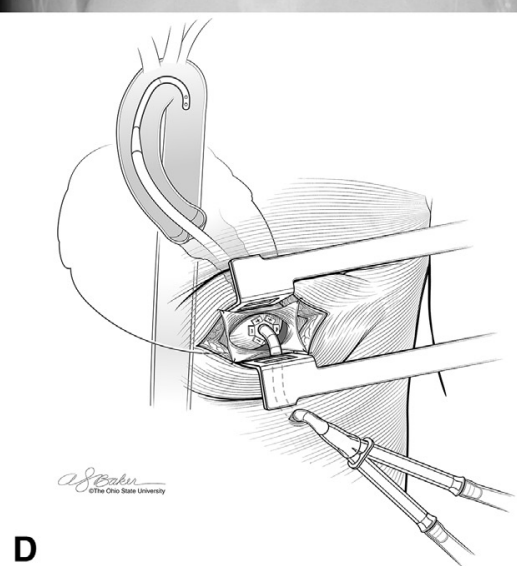

FIGURE 2. A, Intraoperative fluoroscopy after placement of the ProtekDuo cannula through the LV apex through a left anterolateral minithoracotomy incision. Radiologic markers denote the inflow ports positioned in the LV cavity (white arrow) and outflow port positioned in the descending thoracic aorta (black arrow). B, Postoperative chest radiograph. C, Line drawling of pledgeted sutures in the LV apex and dilation of apex using modified Seldinger technique. The guidewire was positioned in the descending thoracic aorta under fluoroscopy. D, Line drawling showing transapical 31-F ProtekDuo cannula tunneled in the interspace below the thoracotomy incision. The pledgeted sutures are tightened with tourniquets (not shown) to secure the cannula and provide hemostasis. The cannula was secured to the skin with suture (not shown).

working with a physical therapist, but unfortunately sustained a hemorrhagic stroke after 26 days of tMCS support. Ultimately, he did not survive this hospitalization.

A similar technique was described by Khalpey and colleagues ${ }^{2}$ in which separate left- and right-sided ProtekDuo cannulas were used to provide biventricular support. Unlike this previous report, we did not modify the distal portion of the left-sided cannula and positioned the outflow port in the descending thoracic aorta. Our patient's history of previous sternotomy and redo sternotomy eliminated central ECMO as a possibility. In addition, his moderate-to-severe aortic insufficiency was a contraindication to peripheral ECMO without LV unloading. Under these circumstances, we choose this method over other tMCS options, such as Impella (Abiomed, Inc, Danvers, Mass) or Impella with peripheral ECMO for several reasons. First, with his aortic pathology, we thought axillary Impella could be more difficult to insert with significant AR and aortic stenosis. The transapical ProtekDuo allowed antegrade placement through the aortic valve with relatively easy positioning of the inflow and outflow ports. With his AR, we thought the ProtekDuo cannula might offload the ventricle more efficiently than Impella. We have experienced hemolysis with Impella, particularly at greater flow settings, which may have been needed with significant AR. In a recent report by Chung and colleagues, ${ }^{3}$ significant hemolysis occurred in $13 \%$ to $26 \%$ of patients supported with axillary Impella support for decompensated heart failure. Lastly, we hoped to eliminate the potential vascular complications while simultaneously providing support that enables ambulation with this technique. The use of hybrid implantation techniques and alternative cannulation strategies enable tMCS as a bridge to decision in patients with severe aortic valve pathology with an increasing emphasis on mobilization to facilitate determination of candidacy for durable advanced therapies.

The authors recognize Anthony S. Baker, CMI, for his exceptional contribution in providing the medical illustrations for the manuscript.

\section{References}

1. Rao P, Khalpey Z, Smith R, Burkhoff D, Kociol RD. Venoarterial extracorporeal membrane oxygenation for cardiogenic shock and cardiac arrest. Circ Heart Fail. 2018;11:e004905. 
2. Khalpey Z, Smith R, Echeverria A, le Tran P, Kazui T. A novel minimally invasive off-pump biventricular assist device insertion technique. J Thorac Cardiovasc Surg. 2016;151:e5-7.
3. Chung JS, Emerson D, Ramzy D, Akhmerov A, Megna D, Esmailian F, et al. A new paradigm in mechanical circulatory support: 100-patient experience. Ann Thorac Surg. 2020;109:1370-7. 